\title{
The globular cluster system of NGC 5846 revisited: colours, sizes and X-ray counterparts ${ }^{\star}$
}

\author{
A. L. Chies-Santos ${ }^{1}$, M. G. Pastoriza ${ }^{1}$, B. X. Santiago ${ }^{1}$, and D. A. Forbes ${ }^{2}$ \\ 1 Departamento de Astronomia, Instituto de Física, UFRGS. Av. Bento Gonçalves 9500, Porto Alegre, RS, Brazil \\ e-mail: ana.leonor@ufrgs.br \\ ${ }^{2}$ Centre for Astrophysics \& Supercomputing, Swinburne University, Hawthorn VIC 3122, Australia
}

Received 15 September 2005 / Accepted 21 April 2006

ABSTRACT

\begin{abstract}
Context. NGC 5846 is a giant elliptical galaxy with a previously well studied globular cluster system (GCS), known to have a bimodal colour distribution with a remarkably high red fraction.

Aims. Here we revisit the central galaxy regions searching for new globular cluster (GC) candidates, and measure magnitudes, colours and sizes for them. We also search for their X-ray counterparts.

Methods. We use archival Hubble Space Telescope WFPC2 images, from which we modelled and subtracted the host light distribution to increase the available sample of GCs. We performed photometry on the central objects, and measured sizes and coordinates for the entire GC system known in this galaxy.

Results. We detect two dozen previously unknown GC candidates in the central regions. Reliable sizes are obtained for about 60 GCs; their typical effective radii are in the range $3-5 \mathrm{pc}$. The largest clusters are located in the central regions. We find $7 \mathrm{X}$-ray counterparts to GCs, most of them in the central region. They are among the most luminous X-ray sources in NGC 5846. They are also optically luminous, compact and belong to the red subpopulation.

Conclusions. The GCS of NGC 5846 is composed of relatively luminous X-ray sources.
\end{abstract}

Key words. galaxies: individual: NGC 5846 - galaxies: star clusters - X-rays: galaxies

\section{Introduction}

NGC 5846 is a giant elliptical galaxy at the center of a large group of galaxies. It has a radial velocity of $1714( \pm 5) \mathrm{km} \mathrm{s}^{-1}$, a distance modulus of $(m-M)=32.32( \pm 0.23)$ and $M_{\mathrm{V}}=-21.57$ (Forbes et al. 1996). It is classified as a transition AGN by Merrifield (2004). The globular cluster system (GCS) of this galaxy was studied by Forbes et al. (1996, 1997a) (FBH1 and FBH2 respectively, or simply FBH when we refer to both papers at once). It was found that NGC 5846 has a much lower specific frequency of globular clusters (GCs) than other dominant ellipticals. On the other hand, similarly to many other luminous early-type galaxies, its GCS has a bimodal colour distribution. The blue and red peaks of the distribution are located at $V-I=0.96$ and 1.17 , respectively, which roughly corresponds to a metalicity of $[\mathrm{Fe} / \mathrm{H}]=-1.2$ and -0.2 . This behaviour is often interpreted as the result of several episodes of star formation or merging contributing to the GCS formation (e.g. Ashman \& Zepf 1992; Forbes et al. 1997b; Côté et al. 1998). Interestingly, FBH found a very high ratio of red to blue GCs. They also observed a smooth radial colour gradient in the sense that the relative fraction of blue GCs increases outwards, something that has also been observed in other galaxies. The central region of NGC 5846, however, is a difficult environment for detecting GCs, even in Hubble Space Telescope (HST) images, given the presence of dust and the high central optical surface brightness. Contrary to other galaxies (e.g. Larsen et al. 2001;

* Tables 3 and 4 are only available in electronic form at the CDS via anonymous ftp to cdsarc.u-strasbg.fr $(130.79 .128 .5)$ or via http://cdsweb.u-strasbg.fr/cgi-bin/qcat?]/A+A/455/453
Jordán et al. 2005), no GC size determinations are so far available for NGC 5846.

Chandra observations of NGC 5846 reveal a complex X-ray morphology of the hot gas. These high resolution images confirm the previously reported similarity between the distribution of X-ray and the $\mathrm{H} \alpha+[\mathrm{NII}]$ emission, indicating that hot and warm gases are linked (Trinchieri \& Goodfrooij 2002). The hard $\mathrm{X}$-ray nucleus is coincident with the radio core observed with the VLA, but is displaced relative to the optical centre (Filho et al. 2004). A population of 41 individual X-ray sources was also observed by Trinchieri \& Goodfrooij (2002), with X-ray luminosities $\left(L_{X}\right)$ in the range of $3 \times 10^{38}-2 \times 10^{39} \mathrm{erg} \mathrm{s}^{-1}$. The luminosity distribution of these sources appears steeper than in any other early type galaxy studied to date. In our Galaxy about $10 \%$ of the luminous X-ray sources are found to be associated with globular clusters, which may harbour X-ray binaries (Verbunt \& Hut 1987). A large fraction of the resolved X-ray point sources in early-type galaxies are likely to be low mass X-ray binaries (LMXBs). Angelini et al. (2001) found that about $70 \%$ of LMXBs in NGC 1399 have a spatial connection with GCs. For NGC 4697 this fraction decreases to 20\% (Sarazin et al. 2001).

The main goal of this paper is to revisit the central regions of NGC 5846 using archival HST images, searching for new GC candidates which may have gone undetected, and investigating their optical properties. We also aim to measure GC sizes and to find any possible link between GCs and X-ray point sources detected by Trinchieri \& Goodfrooij (2002). This paper is organized as follows: in Sect. 2 we summarize the observations and basic data reduction. In Sect. 3 we present the photometry and discuss the colour distribution of the central GCs. In Sect. 4 
Table 1. Journal of observations - HST/WFPC2 images.

\begin{tabular}{cccc}
\hline \hline Rootname & Filter & Pointing & Exposure Time (s) \\
\hline U36J0401T & $F 555 W$ & Central & 900 \\
U36J0402T & $F 555 W$ & & 1300 \\
U36J0403T & $F 814 W$ & & 900 \\
U36J0404T & $F 814 W$ & & 1400 \\
U36J0501T & $F 555 W$ & North & 900 \\
U36J0502T & $F 555 W$ & & 1300 \\
U36J0503T & $F 814 W$ & & 900 \\
U36J0504T & $F 814 W$ & & 1400 \\
U36J0601T & $F 555 W$ & South & 900 \\
U36J0602T & $F 555 W$ & & 1300 \\
U36J0603T & $F 814 W$ & & 900 \\
U36J0604T & $F 814 W$ & & 1400 \\
\hline
\end{tabular}

we determine the sizes of the GCs, assessing the reliability of these measurements. In Sect. 5 we compare the coordinates of the X-ray point sources and the GCs. We present our summary and concluding remarks in Sect. 6.

\section{Observations and data reduction}

Archival HST $V$ and $I$ images from program G0-5920 were used to identify and study in detail GC candidates. The images were obtained with the Wide Field Planetary Camera 2 (WFPC2) in the $F 555 \mathrm{~W}$ and $F 814 \mathrm{~W}$ filters. There are three different pointings, corresponding to 4 images in each pointing, 2 for each filter, resulting in a total of 12 images, see Table 1.

The spatial scale is $0.046^{\prime \prime}$ pixel $^{-1}$ and the field is of $37^{\prime \prime} \times$ $37^{\prime \prime}$ for the planetary camara (PC). For the Wide Field Camera (WFC) the scale is $0.1^{\prime \prime}$ pixel $^{-1}$, corresponding to a $80^{\prime \prime} \times$ $80^{\prime \prime}$ field. The images listed in Table 1 had been previously reduced and used to study the GCS by FBH. These authors measured CCD positions, $V, I$ magnitudes and colours for a sample of a little less than $1000 \mathrm{GC}$. Our main goal in this work was to investigate in more detail the GC properties in the inner regions of NGC 5846. We therefore carried out a careful analysis of the PC images of the central pointing. The initial data reduction process was similar to that of FBH1. We aligned the pairs of individual exposures with each filter and then combined then using the STSDAS task gcombine. In order to identify the GCs in the central region of the galaxy, we carried out detailed surface photometry of the host galaxy. We first built a model of the luminosity distribution of the galaxy using the STSDAS task ellipse for each filter. We then subtracted these models from the $V$ and I PC images that resulted from gcombine. The results of the subtraction reveal a very conspicuous filamentary dust morphology as well as numerous bright compact objects. The sample selection was made by eye using the $V$ subtracted image.

Figure 1 shows the model subtracted image in the $V$-band. The visually selected objects are marked on the image. We selected a total of 196 sources on the subtracted image, 31 of which are in common with FBH. Despite our careful selection, this number is likely to be only a fraction of the total, since it is very complex to determine the exact number of extragalactic globular clusters because of the dificulty to detect them against the bright background of the central regions of their host galaxies.

From the subtracted image we notice that the dust is strongly concentrated in the very center of the galaxy, having an assymetric distribution. In this dusty part of the galaxy the stellar objects appear completely absent. FBH2 suggested that the dust is the result of a past merger or accretion of a gas-rich galaxy and

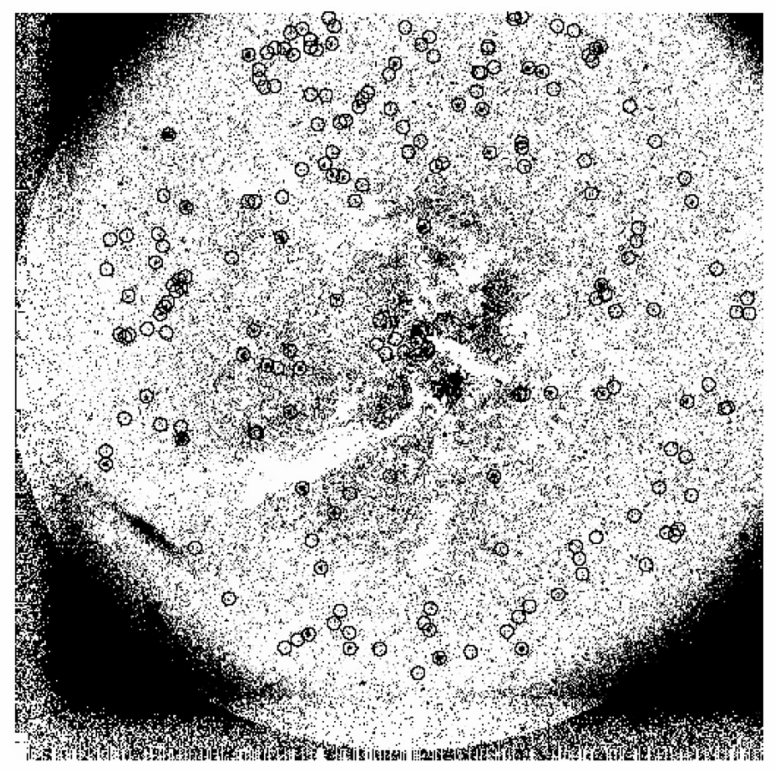

Fig. 1. The result of the subtraction of the model from the gcombined $V$ image in grey scale, $37^{\prime \prime}$ on a side. The eye selected GCs canditades are indicated by circles. North is at about $70^{\circ}$ from the vertical going counterclockwise.

provides the fuel to the compact radio core, which can be seen in both $V$ and $I$ images.

\section{Photometry}

Having CCD positions we then measured magnitudes using the APPHOT task phot, in both gcombined images and the galaxy subtracted ones. In Fig. 2 we show the $V, I$ colour magnitude diagrams (CMD) for all visually selected compact objects obtained from both images. Both CMDs exibit a well defined locus of GCs spanning the range $22.5<V<27.5$ and $0.5<V-I<1.9$. The mean $V-I$ colour of $\sim 1$ is typical of GCs. A total of $68 \mathrm{GC}$ candidates are within these CMD limits. This GC locus shifts blueward at fainter magnitudes as a selection effect, since the sample was visually selected in the $V$ image. Also, the colour dispersion increases substantially for $V>26.5$ due to photometric errors. Notice that the dispersion in colours tends to be smaller in the subtracted images, attesting to the higher photometric precision achieved when subtracted images are used. We therefore used the subtracted images for our photometry and cut the GC sample at $V=26.5$.

In order to have photometric measurements as consistent as possible with those from FBH1, our photometry was carried out in a similar way; we adopted a 2 pixel aperture radius, the aperture corrections to a $0.5^{\prime \prime}$ radius were of 0.39 mag (for $F 555 W$ ) and $0.54 \mathrm{mag}(F 814 W)$. These values were based on Table 2 of Holtzman et al. (1995a). To convert to the standard JohnsonCousins $V, I$ system for a gain ratio of 7 we followed Table 7 of Holtzman et al. (1995b). We finally corrected for Galactic extinction: $A_{V}=0.11$ and $A_{I}=0.05$ (Faber et al. 1989), in order to be consistent with FBH previous work (the Schlegel et al. 1998 values are $A_{V}=0.182$ and $A_{I}=0.107$ ).

Since our final goal is to combine our inner GC sample with the larger sample by FBH we compared our magnitudes in both filters for the common objects. A small offset of $\simeq 0.1 \mathrm{mag}$ was found for both filters. Our final calibrated magnitudes were converted to the FBH1 photometric system by fitting a linear 

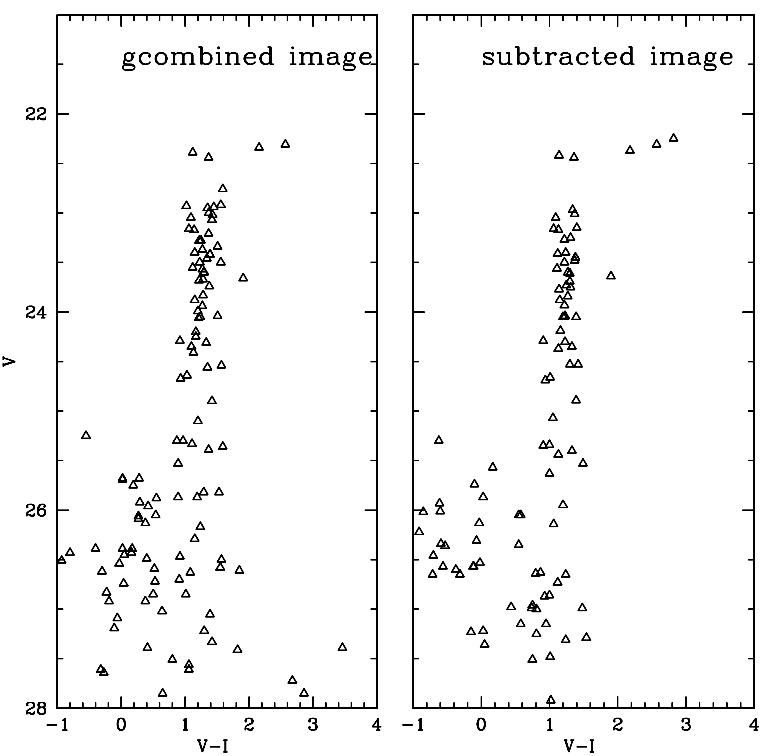

Fig. 2. Left panel: CMD for the gcombined image; right panel: CMD for the subtracted image.

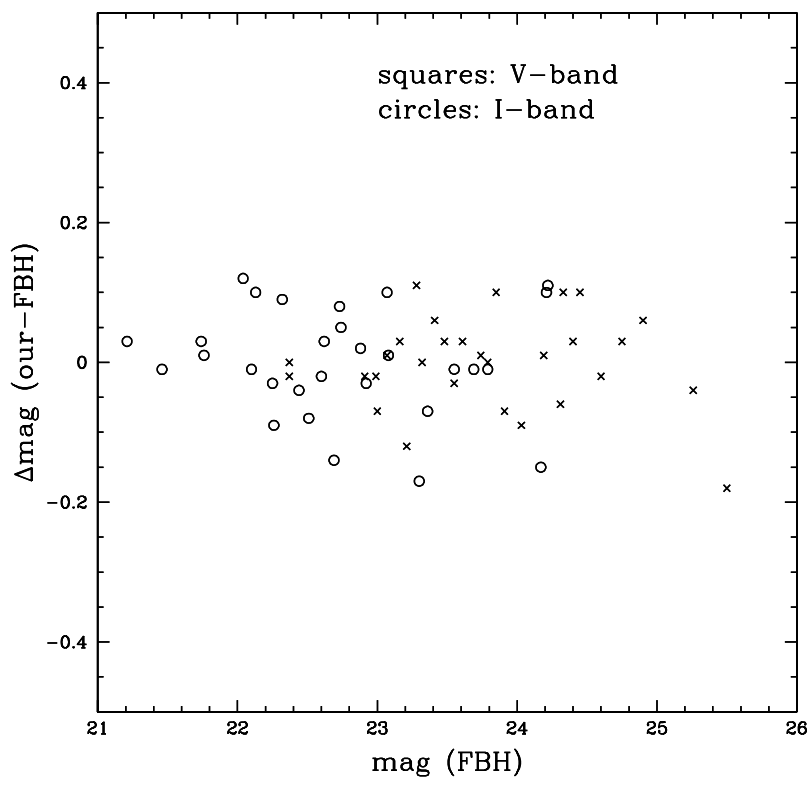

Fig. 3. Photometric differences after comparison between our photometry and that of $\mathrm{FBH}$, after correcting for the fitted linear relation (see text).

relation of the type $m_{\mathrm{FBH} 1}=a m_{\mathrm{our}}+b$, where $m_{\mathrm{FBH} 1}$ is the magnitude as measured by FBH1 and $m_{\text {our }}$ is our corresponding measurement. For the $V$-band coefficients we obtained $a=0.984$ and $b=0.287$; for the $I$-band coefficients we found $a=0.947$ and $b=1.232$. The fits were carried out only for GC candidates with quality photometry $(V<26.5)$. In Fig. 3 we show the residual differences as a function of magnitude for both filters. The scatter of the residuals are \pm 0.07 for the $V$-band and \pm 0.08 for the I-band.

\subsection{Central colour distribution}

Colour distributions are very useful for characterizing GC populations. They have been observed to display a bimodal pattern in large early-type galaxies either in clusters or in the field (Elson \& Santiago 1996 and Rhode \& Zepf 2004). NGC 5846

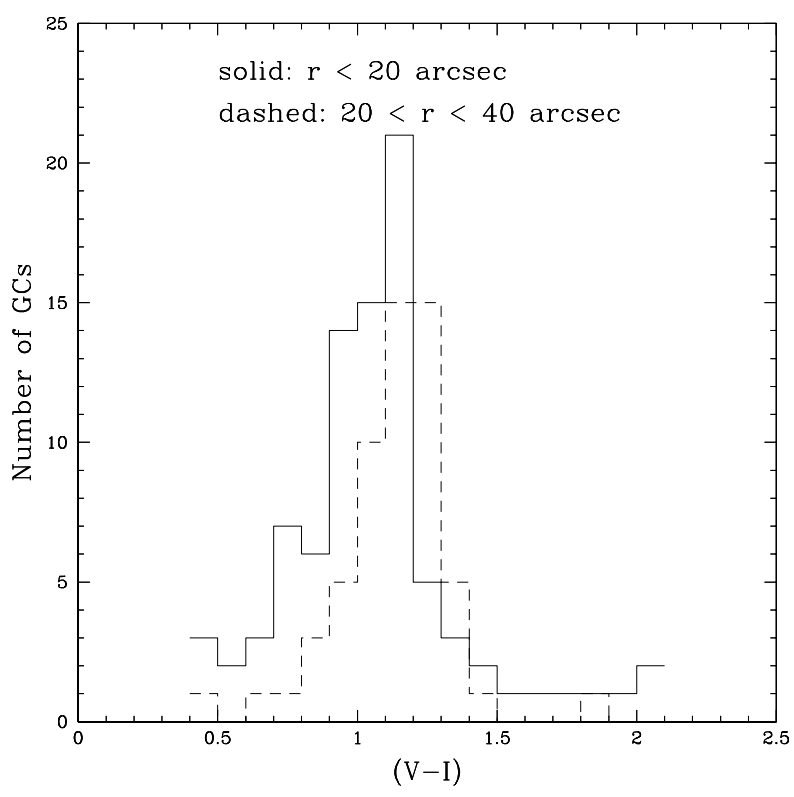

Fig. 4. Colour distributions at the central parts of NGC 5846. Solid line: $r<20^{\prime \prime}$; dashed line: $20^{\prime \prime}<r<40^{\prime \prime}$.

also presents this behaviour, as already pointed out by $\mathrm{FBH}$. These authors also find a variation in the GC colour distribution as a function of galactocentric distance, which they interpret as a metallicity gradient, in the sense that metallicity decreases outwards. Here we take advantage of the increased data sample in the inner regions of the same galaxy and review the GC colour distribution.

Figure 4 shows the colour distribution of GCs with $V<26.5$ for two different rings from the center. Both colour distributions shown are dominated by typical GC colours, ranging from $0.6 \leq(V-I) \leq 1.5$, showing that the GC sample is not strongly contaminated. The inner population is shifted slightly towards bluer colours relative to the one located further out from the center of the galaxy.

One might think that dust could be making the GCs in the outer regions redder. Even though there is dust in this region, as revealed by the lighter spots in Fig. 1 (since its colour is inverted), these spots are clearly more pronounced close to the centre, and therefore should be affecting the inner $\left(r<20^{\prime \prime}\right) \mathrm{GCs}$ more than the ones in the outer regions $\left(20^{\prime \prime}<r<40^{\prime \prime}\right)$. Thus, correction for dust would actually increase the colour difference between the two regions being compared.

The inner regions have a mean $(V-I)=1.17 \pm 0.04$ compared to $(V-I)=1.24 \pm 0.07$ in the next radial bin. Thus the difference $(\delta(V-I)=0.07)$ is small enough to place both regions within the metal-rich GC subpopulation and it is of only marginal statistical significance. We note that the original data of FBH also had a hint for bluer colours at the very center of NGC $5846(r<2 \mathrm{kpc})$, as indicated in their Fig. 5. If real it could be due to a combination of age and/or metallicity effects. The inner GCs could be slightly younger or slightly more metal-poor. It is difficult to test either possibility without high signal-to-noise spectra of these inner GCs, although we may use an SSP model (e.g. Bruzual \& Charlot 2003) to assess age and metallicity effects. Assuming an old population of $12 \mathrm{Gyrs}$ and $[\mathrm{Fe} / \mathrm{H}]=-0.4$ (i.e. similiar to that expected for red GCs), we can investigate the age/metallicity differences needed to reproduce a $\delta(V-I)=0.07$ bluer colour. If the colour difference is purely due to age, then the inner GCs are about 5 Gyrs younger. If purely due to metallicity, 


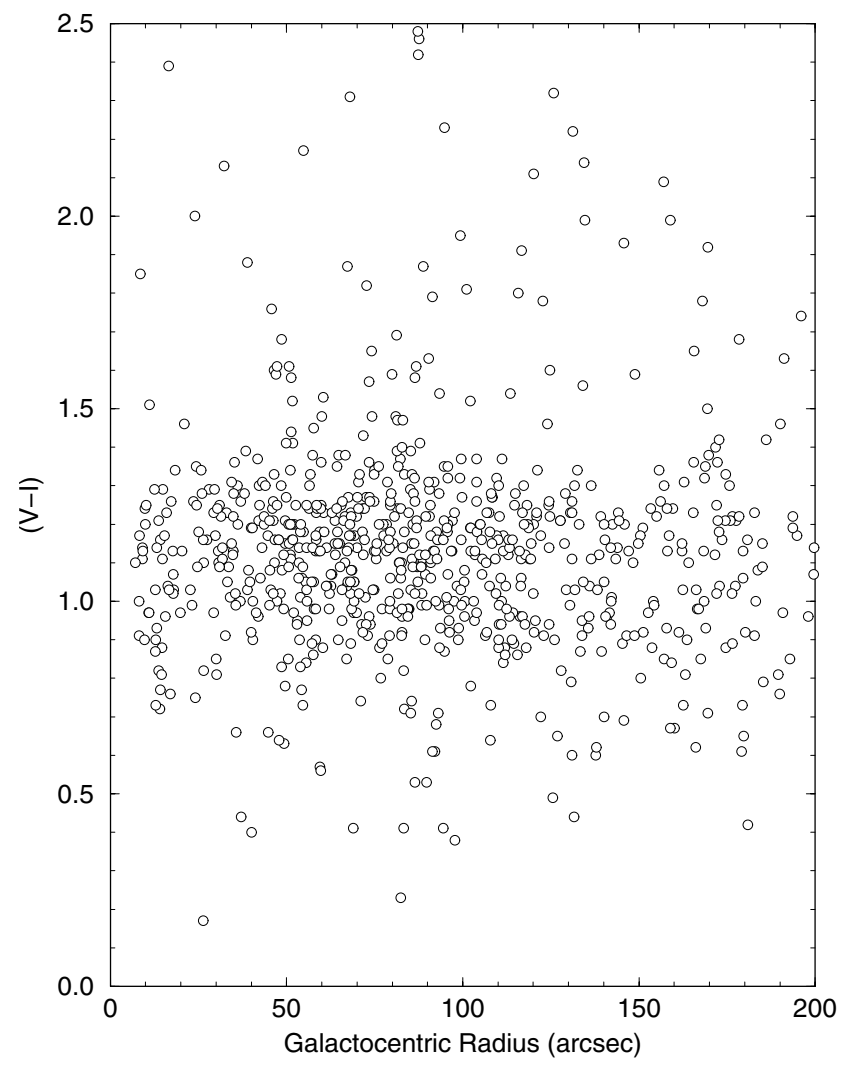

Fig. 5. $(V-I)$ colour of GCs vs Galactocentric radius.

the inner GCs would be more metal-poor by about 0.25 dex. Such large differences would be easily detectable with high S/N spectra. In practice, the bluer colour is likely to be a combination of age and metallicity differences. In Fig. 5 we show a plot of $(V-I)$ as a function of galactocentric radius.

In Table 3 we list the positions and photometry for the entire GCS of NGC 5846.

\section{GCs sizes}

The determination of extragalactic GC sizes is extremely difficult, even in HST data, given their extremely small sizes compared to the extragalactic distances. Larsen \& Brodie (2003) find that the blue GCs are, on average, larger than the red ones. Red GCs are generally smaller than blue GCs by about 20\% (Larsen et al. 2001). In the central parts of galaxies this difference is well pronounced. The effect may, at least in part, arise from a GC size against 3D distance relation, combined with different radial distributions between blue and red GCs (Larsen \& Brodie 2003). Studies of GC sizes are so far largely concentrated on nearby galaxies ( $m-M \simeq 31.5$ or less). In this section we attempt to derive sizes for the NGC 5846 ( $m-M \simeq 32.32)$ GCs.

We use the ISHAPE code described in Larsen (1999). This code convolves the WFPC2/HST point spread function (PSF) with model King profiles in 2 dimensions. The resulting model image is then compared to the real GCs and a $\chi^{2}$ is derived from the fit, along with an estimate of the GC's intrinsic size. We have used an image taken at about the same time as those listed in Table 1 to construct the PSFs for the PC and WFC. We used DAOPHOT.PSF and DAOPHOT.SEEPSF tasks for this purpose. We processed both PC and WFC objects, despite the lower resolution and stronger undersampling of the latter. We tested the sensitivity of the intrinsic sizes to the model concentration
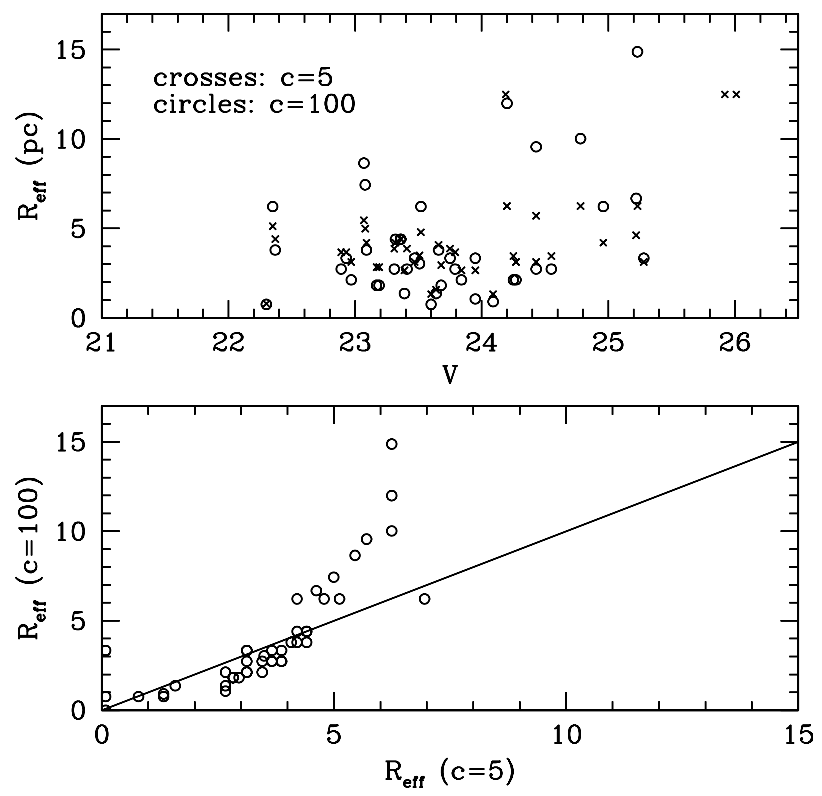

Fig. 6. Upper panel: intrinsic size vs. $V$-band magnitude relation; Lower panel: relation between effective radii as estimated from two extreme King models.

parameter by running ISHAPE for the PC sample both with $c=5$ and $c=100$, which are the extreme values available in the code. In Fig. 6 we show the effective radii $\left(R_{\text {eff }}\right)$ in parsecs as a function of $V$ magnitude for both c values. We also compare the resulting $R_{\text {eff }}$ values. We see that there is no strong trend between size and magnitudes regardless of the King model chosen. However, a slight trend in the sense that brighter GCs are also larger ones is seen brighter than $V \simeq 24$. Also notice that the $R_{\text {eff }}$ values correlate with one another, except at large radii where the $c=5$ models start to saturate. We therefore adopt the $c=100$ model in this paper.

In Fig. 7 we plot the sizes of GCs as a function of $\chi^{2} / \chi_{0}^{2}$, where $\chi_{0}^{2}$ is the result of fitting a point source (delta function) rather than a King profile to the objects. Thus, this ratio quantifies the improvement obtained with the King model relative to a unresolved source. All objects in the sample from the three pointings are shown in the different panels. We note that the larger the $\chi^{2} / \chi_{0}^{2}$ the smaller becomes the radius, as an effect of not resolving the GC from a point source. In particular, objects with $\chi^{2} / \chi_{0}^{2}>0.8$ are essentially unresolved as attested by their systematically small $R_{\text {eff }}$. We therefore cut the sample at $\chi^{2} / \chi_{0}^{2} \leq 0.8$

Next we explore the possibility of a size-colour relation similar to that found by previous authors. In Fig. 8 we plot the sizes of GCs, cut at $\chi^{2} / \chi_{0}^{2}<0.8$, as a function of $(V-I)$ colour, again separating the different pointings in different panels. Even though a relatively small number of GCs is available (especially blue GCs), given our selection criteria, there is no obvious trend in our data for a relation between size and colour. Notice, however, that the larger GCs are seen in the central pointing, specially at the central PC: only a couple of GCs with $R_{\text {eff }}>5$ pc are seen in the South or North pointings, which are located $\sim 2^{\prime}$ from the center.

Finally, the typical size of our resolved GCs is between 3 and $5 \mathrm{pc}$, with only a handful being larger than $10 \mathrm{pc}$. These values are typically larger than the median GC sizes found by Larsen et al. (2001) in their study of nearby galaxies. The difference is likely caused by selection effects, given the larger distance 

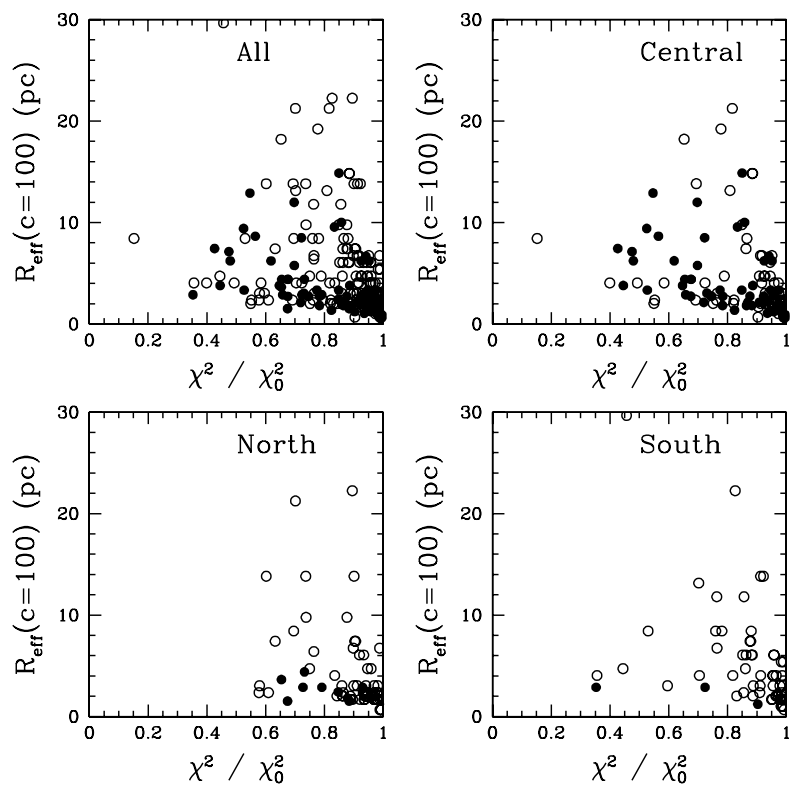

Fig. 7. Size as a function of $\chi^{2} / \chi_{0}^{2}$. Solid symbols represent objects located in the PC chips, whereas open symbols are for WFC chips. Upper left: the entire sample; upper right: central pointing; lower left: north pointing; lower right: south pointing.
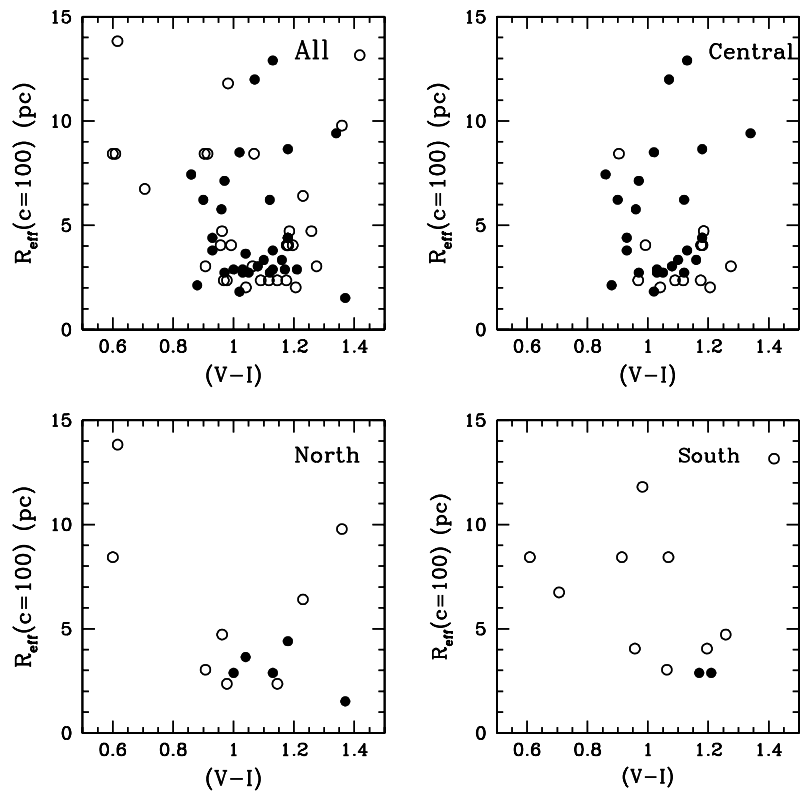

Fig. 8. Size as a function of colour for all 3 individual pointings and for the combined pointing. Open circles are PC GCs and closed ones are WFC GCs.

modulus of NGC 5846. We note that there is still some debate in the literature as to whether such size differences are due to projection effects (e.g. Larsen \& Brodie 2003; Forbes et al. 2005; Spitzer et al. 2006) or mass segregation (Jordan 2004).

Table 4 lists the sizes and related parameters for those GCs which were successfully measured by ISHAPE.

\section{Comparing the GCs and the X-ray point sources}

More than thirty luminous X-ray sources associated with Galactic globular clusters have been observed with Einstein, ROSAT, XMM-Newton and Chandra, since 1975 and up to this

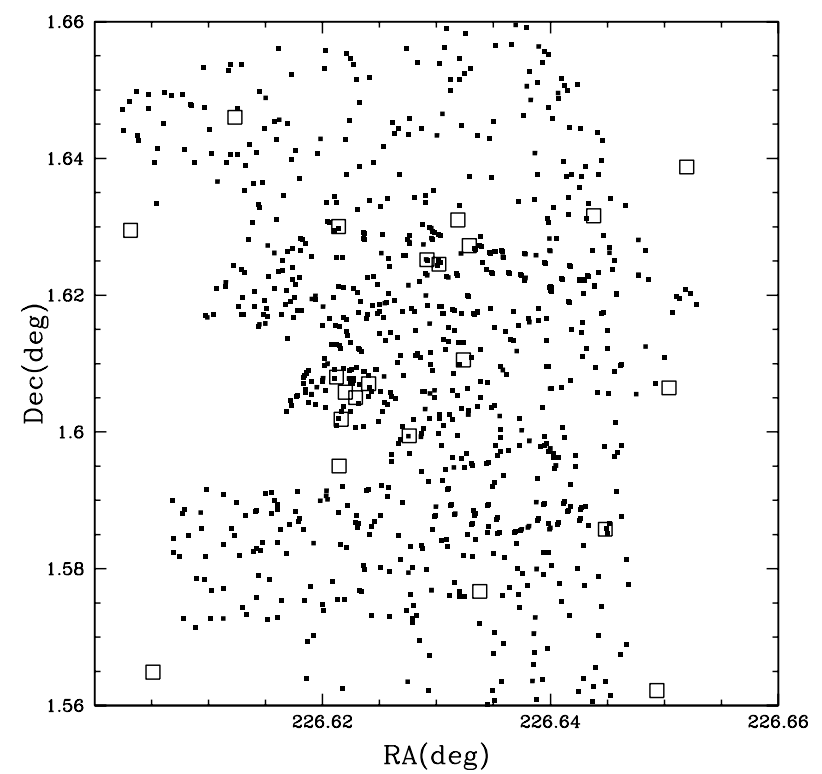

Fig. 9. On-sky map of X-ray sources and GCs in the direction of NGC 5846. The small points represent the GCs and the large squares correspond to the Chandra point sources.

date (Verbunt \& Lewin 2001). On the other hand, high resolution observations made by ROSAT and Chandra in elliptical and spiral galaxies, such as NGC 4697, NGC 4472, M 31 and NGC 4594, reveal X-ray sources associated with GCs. Irwin, Bregman \& Athey (2004) classify ultraluminous X-ray sources (ULXs) as having $L_{\mathrm{X}}=1-2 \times 10^{39} \mathrm{erg} \mathrm{s}^{-1}$ and very ultraluminous X-ray sources (VULXs) as having $L_{X}>2 \times 10^{39} \mathrm{erg} \mathrm{s}^{-1}$. In the early type galaxy NGC 1399 more than two hundred sources were resolved in a $8^{\prime \prime} \times 8^{\prime \prime}$ region, of which 45 were found to be associated with globular clusters and two were found to have $L_{\mathrm{X}}>2 \times 10^{39} \mathrm{erg} \mathrm{s}^{-1}$ (Angelini et al. 2001), therefore being VULXs. In NGC 5846 forty one individual X-ray sources were observed with Chandra X-ray Observatory (Trinchieri \& Goodfrooij 2002). All of them have $L_{X}>3 \times 10^{38} \mathrm{erg} \mathrm{s}^{-1}$. These $L_{\mathrm{X}}$ values are above or close to the Eddington luminosity for a 1.4 solar mass accreting object (Sarazin et al. 2003), suggesting that, if caused by a single X-ray binary, they are accreting onto a black hole, rather than a neutron star. On the other hand, in the case of a globular cluster, more than one LMXBs may be present, mimicking the effect of VULXs.

Here we test the hypothesis that some of these point sources found by Trinchieri \& Goodfrooij (2002) (Table 1 of their paper), are associated with globular clusters in the field of view of HST/WFPC2 studied here. Equatorial coordinates (J2000) were obtained from the WFPC2 images with the STSDAS $x y 2 r d$ IRAF task. This astrometric solution, however, may be systematically offset from the Chandra positions by some unknown amount. Therefore we searched for X-ray counterparts of our GCs at the same time as we found the best estimate for these offsets. We did this by finding the offsets in RA and Dec that maximized the number of matches between optical and X-ray sources. A total of 7 such matches resulted. The corresponding offsets were of $0.8^{\prime \prime}$ in RA and $0.2^{\prime \prime}$ in Dec. These values are consistent with previous astrometric comparisons between HST/WFC2 and Chandra (Jordán et al. 2004; Xu et al. 2005).

We have found GC/X-Ray sources in all three pointings, although most matches are located in the central WFPC2 field. In Fig. 9 we show the on-sky distribution of both optical and X-ray objects, with positions already compensated for the 
Table 2. The globular clusters coincident with Chandra X-ray sources.

\begin{tabular}{cccccc}
\hline \hline Source & $\begin{array}{c}\alpha \\
(\mathrm{J} 2000)\end{array}$ & $\begin{array}{c}\delta \\
(\mathrm{J} 2000)\end{array}$ & $\begin{array}{c}V \\
(\mathrm{mag})\end{array}$ & $\begin{array}{c}(V-I) \\
(\mathrm{mag})\end{array}$ & $\begin{array}{c}L \mathrm{Cx} \\
\left(\mathrm{erg} \mathrm{s}^{-1}\right)\end{array}$ \\
\hline 8 & $15: 06: 29.15$ & $+01: 36: 28.7$ & 22.8 & 1.12 & $9.32 \times 10^{39}$ \\
9 & $15: 06: 29.19$ & $+01: 37: 47.9$ & 25.23 & 2.42 & $4.2 \times 10^{38}$ \\
11 & $15: 06: 29.25$ & $+01: 36: 06.5$ & 23.39 & 1.17 & $1.77 \times 10^{39}$ \\
15 & $15: 06: 30.68$ & $+01: 35: 57.8$ & 23.62 & 1.29 & $4.2 \times 10^{38}$ \\
16 & $15: 06: 31.05$ & $+01: 37: 30.4$ & 23.23 & 1.12 & $1.22 \times 10^{39}$ \\
17 & $15: 06: 31.31$ & $+01: 37: 28.1$ & 24.32 & 1.26 & $6.5 \times 10^{38}$ \\
24 & $15: 06: 34.8$ & $+01: 35: 08.6$ & 23.16 & 1.15 & $7.8 \times 10^{38}$ \\
\hline
\end{tabular}

offsets mentioned above. We find that the matched objects tend to concentrate to the inner parts of the galaxy. In Table 2 we list the main properties of these GC/X-ray objects. The listed positions are those from Trinchieri \& Goodfrooij (2002). Their colours show that they belong to the red population, with no exception. This result is in agreement to what Angelini et al. (2001) found for NGC 1399. We also notice that the $V$ magnitudes of 5 matched objects are brighter than 23.6, which means that the X-ray emitting GCs are usually brighter than average, as previously found by Sarazin et al. (2003) and Kundu et al. (2002) for other luminous ellipticals. Considering their intrinsic sizes, only three of the matched objects were resolved with the ISHAPE code (Sect. 4), but 2 of them were marginally resolved; all three are smaller than $R_{\mathrm{eff}}=2.8 \mathrm{pc}$, being therefore bright but compact GCs.

In terms of X-ray properties, several of our matched GCs lie among the most luminous objects in the host galaxy. Source 16 is a ULX and sources 17 and 24 have measured $L_{\mathrm{x}}$ close to $10^{39} \mathrm{erg} \mathrm{s}^{-1}$, being among the $25 \%$ brightest $\mathrm{X}$-ray sources in NGC 5846. They are therefore likely to be either a single binary system accreting onto a black hole or several LMXBs at or near the Eddington luminosity, as discussed in Angelini et al. (2001). Besides, the X-ray sources 8 and 11 have been classified by Trinchieri \& Gooudfrooij (2002) as extended regions. Alternatively, they could be composed of several overlaping sources having different origins. Because they have total luminosity of $L_{\mathrm{x}}>2 \times 10^{39} \mathrm{erg} \mathrm{s}^{-1}$, the corresponding GC match may still be a ULX.

We note that NGC 5846 has been described as a complex X-ray object by Filho et al. (2004). They find a coincidence between the VLA radio core and the X-ray nucleus of the galaxy; this latter position is where source number 12, according to classification of Trinchieri \& Goodfrooij (2002), is located. Notice that this is one among several other luminous X-ray sources. Also, we observe that the position of source 12 is not coincident with the optical center.

Since VULXs are found to be associated with late rather than early-type galaxies, NGC 5846 seems to be an exception along with NGC 1399.

\section{Summary and concluding remarks}

With $V$ and $I$ WFPC2 images taken from the Hubble Space Telescope public archive we have detected 68 central globular clusters within the PC ( $r \leq 2 \mathrm{kpc}) ; 23$ of these are previously undetected objects. We combined our inner sample with the larger data set by FBH and revisited the properties of this central sample. We have determined the coordinates and photometry for all GCs in the combined sample and also attempted to measure their intrinsic sizes. For several dozens, useful estimates of the effective radius were obtained, especially in the
PC image. This information is given in two electronic tables. We also searched for X-ray counterparts to the GCs in the Chandra sample of Trinchieri \& Goodfrooij (2002).

Our main results are:

1. The colour distribution shows a hint of becoming bluer in the very central galaxy regions.

2. Most GCs with reliable size estimates have $R_{\mathrm{eff}}>3 \mathrm{pc}$; larger GCs tend to be seen in the central region of the host galaxy. In fact, only a few GCs with $R_{\text {eff }}>5$ pc are located outside the central pointing. This is opposite to the behaviour found by van den Bergh et al. (1991) for the Milky Way GCs. We note however, that all three early-type galaxies with extensive spatial coverage in the sample of Larsen et al. (2001) show an increase in median GC $R_{\text {eff }}$ in the most central radial bin, especially for the blue sub-population. The central pointing also has the largest total number of GCs, so if the large GCs are just the tail of the GC size distribution, then one would expect an statistical excess of these large GCs where there are more GCs in general. However, the excess of large GCs in the central pointing remains after we correct for this effect: the outer fields have about 0.4 times the number of GCS in the central pointing, but they have fewer GCs with $R_{\text {eff }}>5$ pc.

3. No clear evidence for a size-colour relation is found. This result does not seem to agree with the findings of Larsen et al. (2001), who observed that the blue clusters are generally larger than the red ones by about $20 \%$. However this is possibly a selection effect as NGC 5846 is more distant their more nearby galaxies (for which smaller intrinsic sizes could be measured) and our sample has very few blue GCs.

4. From a positional match between optical and X-ray coordinates, we found $7 \mathrm{GC} / \mathrm{X}$-ray matches, most of which are located in the central parts. Optically, they tend to be bright $(V \leq 23.5)$ and compact, since only one was clearly resolved. All of them have $(V-I)>1.1$, which places them as members of the red sub-population. Their X-ray luminosities are also among the highest in the Chandra sample, with 3 of them being among the $25 \%$ most luminous and 2 other matches lying in complex central regions with $L_{\mathrm{X}}>10^{39} \mathrm{erg} \mathrm{s}^{-1}$.

The X-ray emitting GCs are typically more luminous in X-rays than those found in other early-type galaxies. For instance Angelini et al. (2001) found that $70 \%$ of the X-ray point sources in the direction of NGC 1399 were located within GCs, but almost all of them have $L_{\mathrm{X}}<10^{39} \mathrm{erg} \mathrm{s}^{-1}$. Our lower detection rate of GC/X-rays in comparison to theirs, associated with a higher fraction of luminous sources, suggests that the GCS of NGC 5846 is composed of relatively few, although very luminous X-ray sources.

This conclusion also seems to hold when NGC 5846 is compared to other ellipticals, either very luminous ones, such as NGC 4472, or more typical, such as NGC 4697 (Kundu et al. 2002; Sarazin et al. 2000). At the high end of the $L_{X}$ luminosity function, Trinchieri \& Goodfrooij (2002) found 3 ULXs and 4 extended regions, with high total $L_{X}$ out of 41 X-ray point like sources, where around 2 would be expected to be foreground/background sources (Irwin et al. 2004). NGC 5846 displays an extended and complex structure in X-ray emission, described by Filho et al. (2004), something that is also observed in other early-types, like NGC 1600 (Sivakoff et al. 2004). We are currently analysing images obtained from the New Technology Telescope (NTT) and HST archive of NGC 1600 in order to 
check if there is any similarity to NGC 5846. A correlation between the overall galactic X-ray properties and the GCS would provide useful additional constraints to the processes that govern the formation of both GCs and their host galaxy.

Acknowledgements. We acknowlodge the financial support of $\mathrm{CNPq}$ and the comments and suggestions of an annonymous referee. DF thanks the ARC for financial support. We thank J. Irwin for comments and suggestions that helped improve the paper.

\section{References}

\section{References}

Angelini, L., Loewenstein, M., \& Mushotzky, R. F. 2001, ApJ, 557, L35

Ashman, K. M., \& Zepf, S. E. 1992, ApJ, 384, 50

Bruzual, G., \& Charlot, S. 2003, MNRAS, 344, 1000

Côté, P., Marzke, R. O., \& West, M. J. 1998, ApJ, 501, 554

Elson, R. A. W., \& Santiago, B. X. 1996, MNRAS, 280, 971

Faber, S. M., Wegner, G., Burstein, D., et al. 1989, ApJS, 69, 763

Filho, M. E., Fraternali, F., Markoff, S., et al. 2004, A\&A, 418, 429

Forbes, D. A., Brodie, J. P., \& Huchra, J. 1996, AJ, 112, 2448

Forbes, D. A., Brodie, J. P., \& Huchra, J. 1997a, AJ, 113, 887

Forbes, D. A., Brodie, J. P., \& Grillmair 1997b, AJ, 113, 1652

Forbes, D. A., et al. 2005, MNRAS, in press
Hempel, M., \& Kissler-Patig, M. 2004, A\&A, 428, 459

Holtzman, J. J., Hester, J. J., Casertano, S., et al. 1995a, PASP, 107, 156 Holtzman, J. J., Burrows, C. J., Casertano, S., et al. 1995b, PASP, 107, 1065 Irwin, J. A., Bregman, J. N., \& Athey, A. E. 2004, ApJ, 601, L143I Jordán, A. 2004, ApJ, 613, L117

Jordán, A., Côté, P., Ferrarese, L., et al. 2004, AJ, 613, 279

Jordán, A., Côté, P., Blakeslee, J. P., et al. 2005, ApJ, 634, 1002

Kundu, A., Maccarome, T., \& Zepf, S. 2002, ApJ, 574, L5

Larsen, S. S. 1999, A\&AS, 139, 393

Larsen, S. S., \& Brodie, J. P. 2003, ApJ, 593, 340

Larsen, S. S., Brodie, J. P., Huchra, J. P., Forbes, D. A., \& Grillmair, C. J. 2001, AJ, 121, 2974

Merrifield, M. R. 2004, MNRAS, 353, L13

Rhode, K. L., \& Zepf, E. Z. 2004, AJ, 127, 302

Sarazin, C. L., Irwin, J. A., \& Bregman, J. N. 2000, ApJ, 544, 101

Sarazin, C. L., Irwin, J. A., \& Bregman, J. N. 2001, ApJ, 556, 533

Sarazin, C. L., Kundu, A., Irwin, J. A., et al. 2003, ApJ, 595, 743

Schlegel, D. J., Finkbeiner, D. P., \& Davis, M. 1998, ApJ, 500, 525

Sivakoff, G. R., Sarazin, C. L., \& Carlin, J. L. 2004, ApJ, 617, 262

Spitzer, L., et al. 2006, AJ, submitted

Trinchieri, G., \& Goodfrooij, P. 2002, A\&A, 386, 472

van den Bergh, S., Morbey, C., \& Pazder, J. 1991, ApJ, 375, 594

Verbunt, F., \& Hut, P. 1987, in The Origin and Evolution of Neutron Stars, ed. D. Helfand, \& J.-H. Huang (Dordrecht: Reidel), IAU Symp., 125, 187

Verbunt, F., \& Lewin, W. H. G. 2004 [arXiv:astro-ph/0404136]

Xu, Y., Xu, H., Zhang, Z., et al. 2005, ApJ, 631, 809 